\title{
An Approach for Utility Pole Recognition in Real Conditions
}

\author{
Alejandro Israel Barranco-Gutiérrez, Saúl Martínez-Díaz, \\ and José Luis Gómez-Torres \\ Instituto Tecnológico de La Paz, B.C.S., México \\ abarrancog0900@ipn.mx, \\ saulmalitlp.edu.mx, \\ joseluismx33@gmail.com
}

\begin{abstract}
In this work, we propose an approach for utility pole recognition in real conditions based on color, shape and photometric stereo vision, by using conventional low cost cameras. This subsystem is part of an automatic path planning system for a maintenance robot, which repairs the cable connections in electrical poles. This method could be used in applications requiring object recognition in outdoor environments. The challenges facing this approach include extreme solar illumination, the confusion between telephone poles, cable TV, in columns of buildings, trees, street lights, and tilt between the ground and the pole. The experiments of this recognition system shows satisfactory results under different solar illuminations, different distances between the post and the cameras, different inclinations of pole with respect to the ground, occlusions of the pole and location of the utility pole from cameras system. Results were totally satisfactory with $100 \%$ effectiveness in a range of 5\% to $95 \%$ with respect to the H component of the HSV scheme. The proposed method recognizes and locates utility poles with respect to the stereo vision system.
\end{abstract}

Keywords: Color segmentation, Stereo vision, Utility pole, Object shape, Solar lighting.

\section{Introduction}

One reason why the robots were created is for the execution of any dangerous task for humans. An important application of the robots could be the repair of cable connections in utility poles (UP). For this task it is necessary to recognize the UP, cables, screws, nuts, thus dimensions and locations [1]. This work begins specifically with the recognition of Mexican concrete UP installed by CFE (Mexican Electricity Federal Company) and to extend it to installations by other companies. There are many techniques for object recognition in controlled environments; unfortunately the UP's are placed in open areas with different backgrounds and, in many cases, under extreme conditions of illumination. The lighting, the distance between the target and the camera, the inclination of the ground on UP and the texture of the pole are very important aspects to be considered in the recognition process. For the automatic repair of connections, it is necessary to set a vision system which recognizes the above mentioned objects and estimates its location, Figure 1.illustrates the configuration of vision and robot system to emulate the cable repairing on a Japanese UP. 


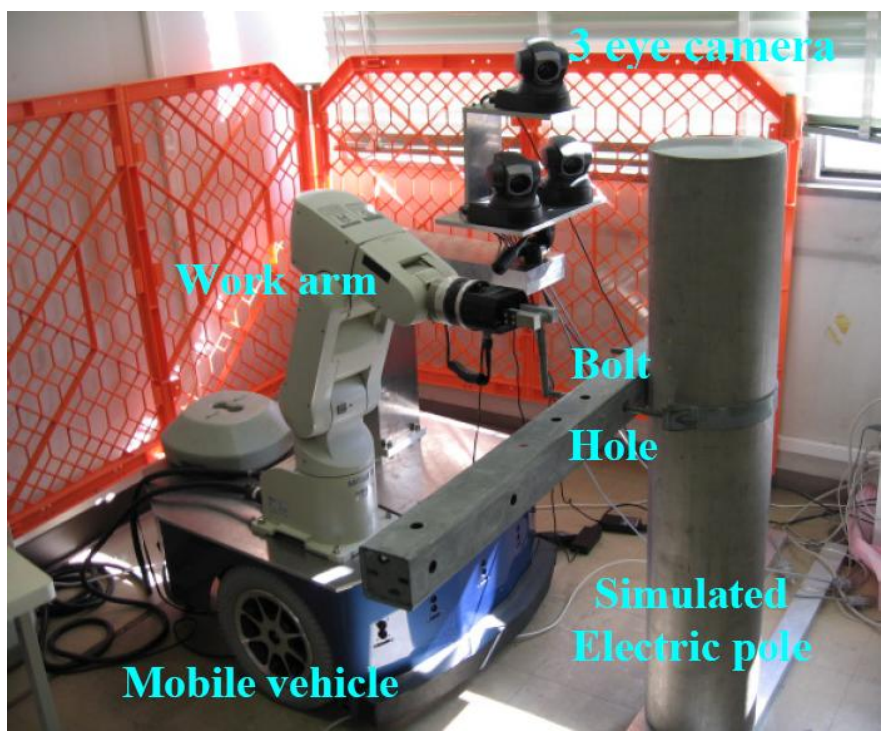

Fig. 1. Robot and cameras mounted to emulate repairing connections with Japanese utility pole

In this document a proposal for UP recognition system is presented with satisfactory results, which is the basis for designing a highly accurate methodology. The global idea consists in three steps:

- Color segmentation

- Shape recognition and inclination estimation

- Electrical pole diameter measurement by stereo vision system.

\section{Related Work}

The set of techniques currently existing for object recognition is very large. In contrast, there are few specific proposals to solve the problem of the UP recognition, which is complicated because there are many objects on the street such as PVC pipes, phone poles, transit signals and cable TV poles. Reference [2] proposes an approach for automatically inputting handwritten Distribution Facility Drawings (DFD) and their maps into a computer, by using the Facility Management Database (FMD). The recognition method makes use of external information for drawing/map recognition. It identifies each electric-pole symbol and support cable symbol on drawings simply by consulting the FMD, but the system requests a manual feedback from the operator in online stage. Reference [3] presents an algorithm for the recognition of similar electrical poles from an aerial image by detecting the pole shadow. One pole is used as a template (already identified by a human operator) for the algorithm. The algorithm includes feature extraction, candidate position determination, and elimination of redundant candidates. 
This work references the ideas from other works in similar sense, the paper [4][5]details, although the color distribution changes under different lighting conditions, some aspects of its structure turn out to be invariants. Also reference [6][7] explains that the sky has always been the crucial element in modeling the background of an outdoor scene. The position of the sun during the day gives a different impact on the sky color. The method presented in [8] is used to compare our method although the acquisition system is different.

\section{$3 \quad$ Proposed Methodology}

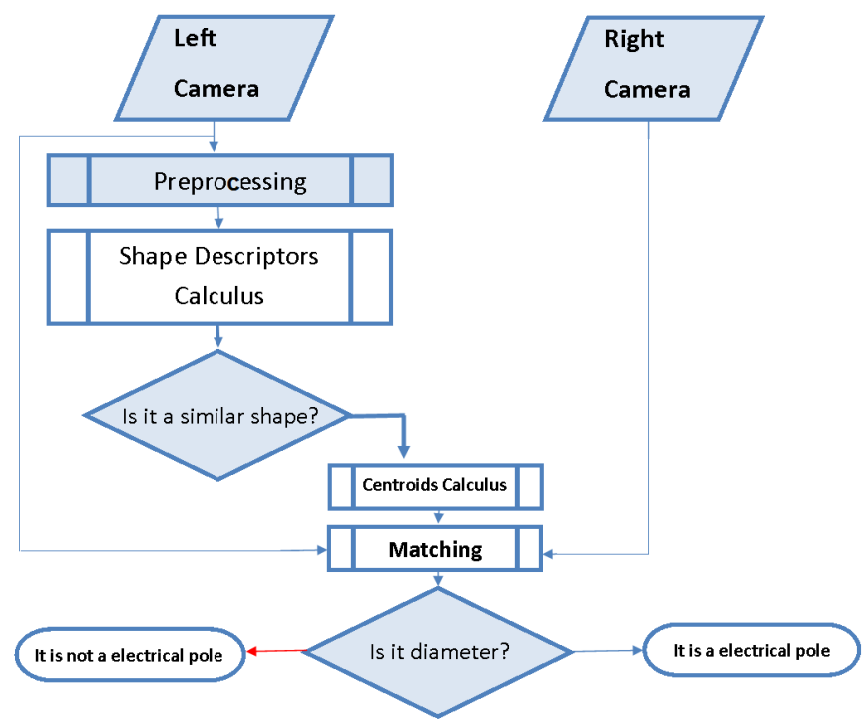

Fig. 2. Flow diagram of proposed method

The proposed methodology is shown in figure 2. The preprocessing stage consists of the following steps:

- RGB to HSV images conversion

- Color filtering

- $\quad$ Borders erasing

- Component Labeling

The HSV is used because it separates the illumination from color components, $\mathrm{H}$ component describe the color of pixels, $\mathrm{V}$ component the illumination and the $\mathrm{S}$ component the saturation of color. This scheme is more sensitive when lighting is reduced than when it is augmented, respect to the center of $\mathrm{V}$ axis because the illumination depends of $\mathrm{R}, \mathrm{G}$ and $\mathrm{B}$ components when the illuminations is on pure white-black axis $\boldsymbol{I}_{\boldsymbol{R}}=\boldsymbol{I}_{\boldsymbol{G}}=\boldsymbol{I}_{\boldsymbol{B}}=\boldsymbol{I}$ as illustrate the Figure 3 . 


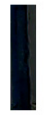

$\mathrm{H}$
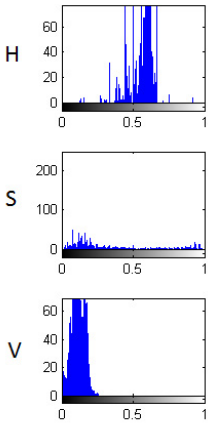

$-30$
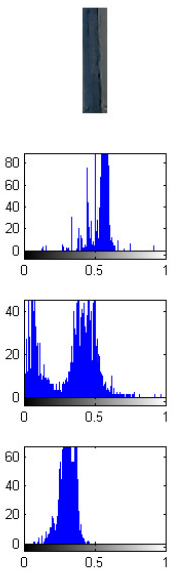

$-20$
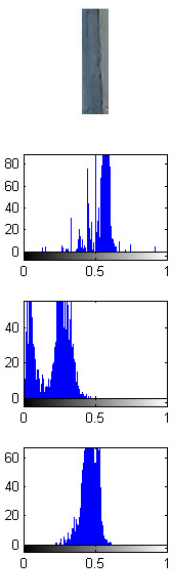

$-10$
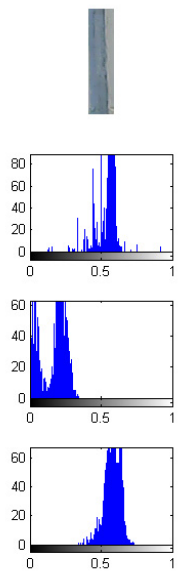

$+0$
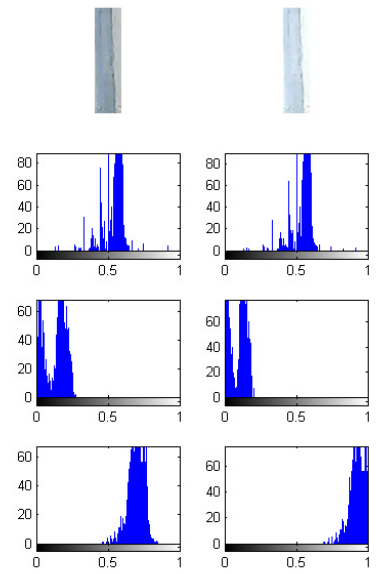

$+30$

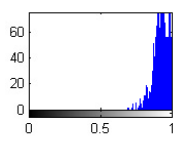

$+60$

Fig. 3. The histograms in HSV scheme of a piece of utility pole under different illuminations $(\mathrm{I}=-30, \mathrm{I}=-20, \mathrm{I}=-30, \mathrm{I}=0, \mathrm{I}=+30$ and $\mathrm{I}=+60$ )

The color is invariant to the lighting in the range where the lighting is not extreme. In Figure 3 are shown the $\mathrm{H}$ histograms in different illumination conditions, when the luminosity on the pole is extreme the histogram of $\mathrm{H}$ component is deformed. Hence we can work on a wide range where the lighting or darkness is not extreme. $\mathrm{V}$ component discriminates the extreme cases using only the range between 5\%-95\% of its values. Respect to the UP color, the $\mathrm{H}$ component is used to identify it, the limits of color are defined by the mean of inferior and superior limits $\left[\overline{\boldsymbol{l}_{\boldsymbol{l}}}, \overline{\boldsymbol{l}_{\boldsymbol{s}}}\right]$ for each $\mathrm{H}_{\mathrm{i}}$ histogram sampled. It obtains its mean and standard deviation of $\mathrm{H}_{\mathrm{i}}$, inferior limit $\boldsymbol{l}_{\boldsymbol{i}}=\overline{\boldsymbol{x}}_{\boldsymbol{\imath}}-\mathbf{3} \boldsymbol{\sigma}_{\boldsymbol{i}}$ and superior limit $\boldsymbol{l}_{\boldsymbol{s}}=\overline{\boldsymbol{x}}_{\boldsymbol{\imath}}+\mathbf{3} \boldsymbol{\sigma}_{\boldsymbol{i}}$ as considers Tchebycheff [9].

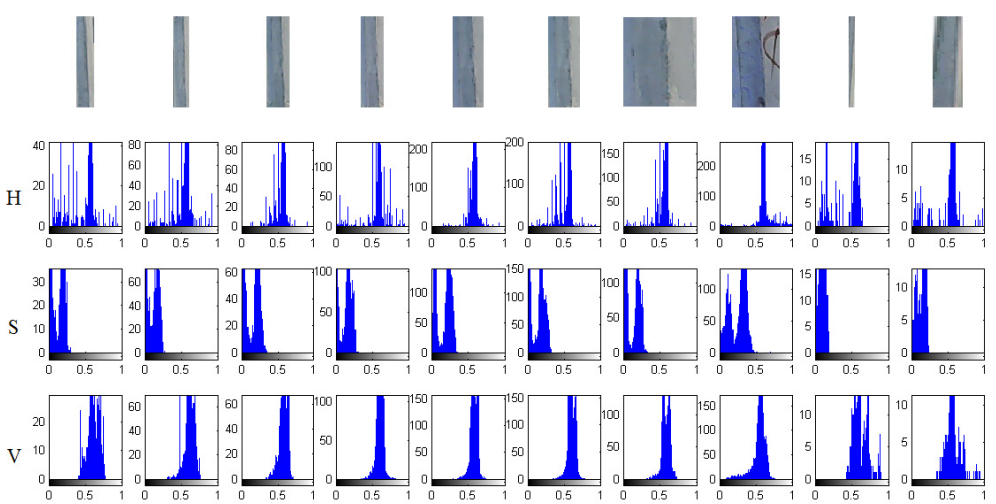

Fig. 4. The histograms of different utility poles with the same solar illumination 
Once the left images are segmented by color, the border detection [10] is necessary to find limits between segments of concrete color but of different bodies, for example building columns, walls, other poles, sidewalks and ground. A simple "AND" operation is implemented to delimit the body silhouettes, between color segmented image and the complement of edge image. Then the components labeling process is executed. For the training stage, the pole segments are selected to teach a Neural Network the UP shape through classic Hu moments [11] invariants to scale, rotations and translations. Finally it measure the pole diameter (in our case is from $25 \mathrm{~cm}$. at the lowest to $19 \mathrm{~cm}$. in the upper).

The features provided to the $\mathrm{NN}$ are 8, the seven $\mathrm{Hu}$ invariants and segment area. The latter because the UP has rectangular shape when it is near to the cameras and it has a line shape when it is away.

To measure the pole diameter a heuristic is implemented (probably only valid to this problem) as part of feature detection for 3D recognition [12][13]. The line equation that approximates the inclination of the UP is estimated using least squares [14] as shown Figure 5.

$$
\begin{gathered}
\sum_{i=1}^{n} y_{i}=n a+b \sum_{i=1}^{n} x_{i} \\
\sum_{i=1}^{n} x_{i} y_{i}=a \sum_{i=1}^{n} x_{i}+b \sum_{i=1}^{n} y_{i}^{2} \\
f\left(x_{i}\right)=\widehat{a}+\widehat{b} x_{i}
\end{gathered}
$$

The perpendicular line is simply calculated by $\mathbf{m}_{\mathbf{1}} \mathbf{m}_{\mathbf{2}}=-\mathbf{1}$ relation.

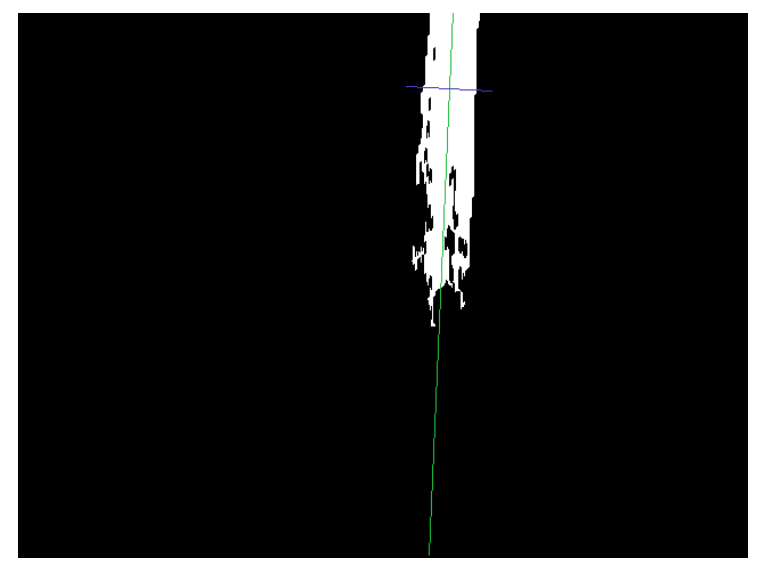

Fig. 5. Estimation of silhouette inclination and the descriptors points of utility pole diameter

The right point results from intersection of a perpendicular line that passes on centroid from left to right UP edges. The equations of left and right edges are 
estimated in the same way as (6) and they are intersected with (7) to find the left and right extremes of UP diameter $\boldsymbol{p}_{\boldsymbol{l}}{ }^{\boldsymbol{l}}=\left(\boldsymbol{x}_{\boldsymbol{l}}{ }^{\boldsymbol{l}}, \boldsymbol{y}_{\boldsymbol{l}}{ }^{\boldsymbol{l}}\right)$ and $\boldsymbol{p}_{\boldsymbol{r}}{ }^{\boldsymbol{l}}=\left(\boldsymbol{x}_{\boldsymbol{r}}{ }^{\boldsymbol{l}}, \boldsymbol{y}_{\boldsymbol{r}}{ }^{\boldsymbol{l}}\right)$ in the left image, where the superscript indicates the left or right camera.

$$
y-\bar{y}=\frac{-1}{\widehat{b}}(x-\bar{x})
$$

As soon as the two points are detected, normalized correlation on gray scale image is employed with the purpose of match corresponding points of each image of stereo vision system $\boldsymbol{p}_{\boldsymbol{l}}{ }^{\boldsymbol{r}}=\left(\boldsymbol{x}_{\boldsymbol{l}}{ }^{\boldsymbol{}}, \boldsymbol{y}_{\boldsymbol{l}}{ }^{\boldsymbol{r}}\right)$ and $\boldsymbol{p}_{\boldsymbol{r}}{ }^{\boldsymbol{r}}=\left(\boldsymbol{x}_{\boldsymbol{r}}{ }^{\boldsymbol{r}}, \boldsymbol{y}_{\boldsymbol{r}}{ }^{\boldsymbol{r}}\right)$. With the information of the two detected points the diameter is computed. The method uses a calibrated stereo vision system for dimensional verification of electric pole diameter. The Zhang's calibration method brings a calibration matrix and distortion coefficients [15][16], these data are necessary to triangulation of points that provides the location of them, relative to one camera. Stereo calibration helps to get the vector between the principal points of both cameras. The next step is triangulation between the same interest points on different images. Finally, with the diameter information, the system is able to decide if an electric pole is present in the tested image.

$$
(d x, d y, d z)=\operatorname{triangulation}\left\{p_{l}^{l}, p_{l}^{r}\right\}-\operatorname{triangulation}\left\{p_{r}{ }^{l}, p_{r}^{r}\right\}
$$

$$
18 \mathrm{~cm} .<\sqrt{d x^{2}+d y^{2}+d z^{2}}<26 \mathrm{~cm}
$$

(9) yields a tolerance of $1 \mathrm{~cm}$. To express the UP centroid in robot reference system the triangulation of centroid coordinates is necessary to insert them in the vision-robot transformation [16].

\section{$4 \quad$ Experimental Results}

In this section we show experimental results obtained with the proposed method. The database is composed by 200 different photos of real UP's on the street at different distances between the cameras and our target (100 photos for left side and 100 for right side). For the first step, stereo images are taken from conventional cameras with $432 \times 576$ pixels of resolution in RGB as shown Figure 6 each image is converted to HSV scheme [17].
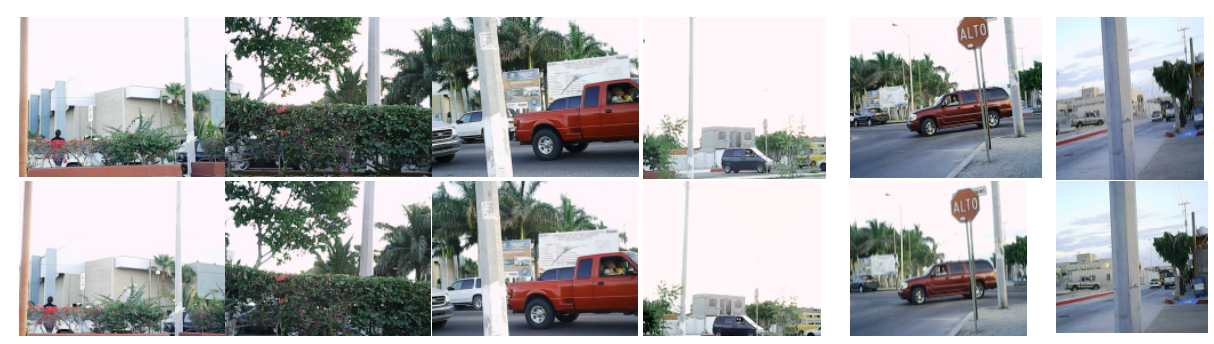

Fig. 6. Left (up row) and right photos of utility poles used in different conditions 
Everyone can access the database on the web address: http://posgrado.itlp.edu.mx/ barranco/ and experiment with this information.

The color filter in this case is implemented with $\overline{\boldsymbol{x}}=\mathbf{0 . 5 8 5}$ and $\boldsymbol{\sigma}=\mathbf{0 . 0 4 5}$ on $\mathrm{H}$ component of HSV scheme, according with $\mathrm{H}$ component histograms of utility pole, to get segmented images as shown in Figure 8. The classification is implemented by a Neural Network of 10 hidden layers and the decision threshold at 0.97. Figure 7 shows that the NN easily discriminate between classes with small error, without confusion, from 40 UP shapes and 50 not UP shapes. It indicates that descriptors were well chosen.

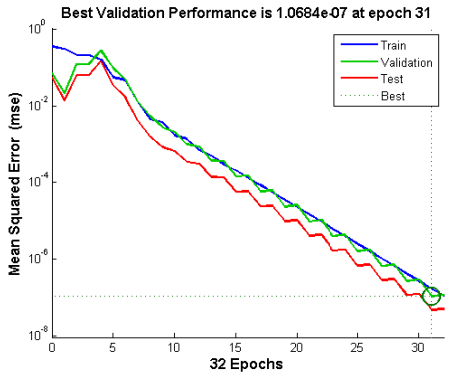

(a)

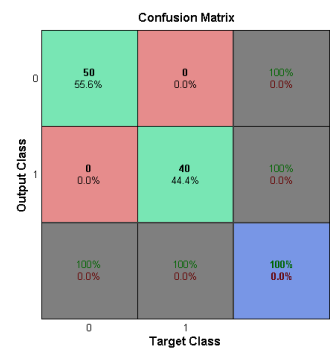

(b)

Fig. 7. (a) Performance of NN training. (b) Confusion matrix in training

The training stage uses 40 photos with all segments that include at least one UP and many not UP segments. The user chose some of them to train the NN.

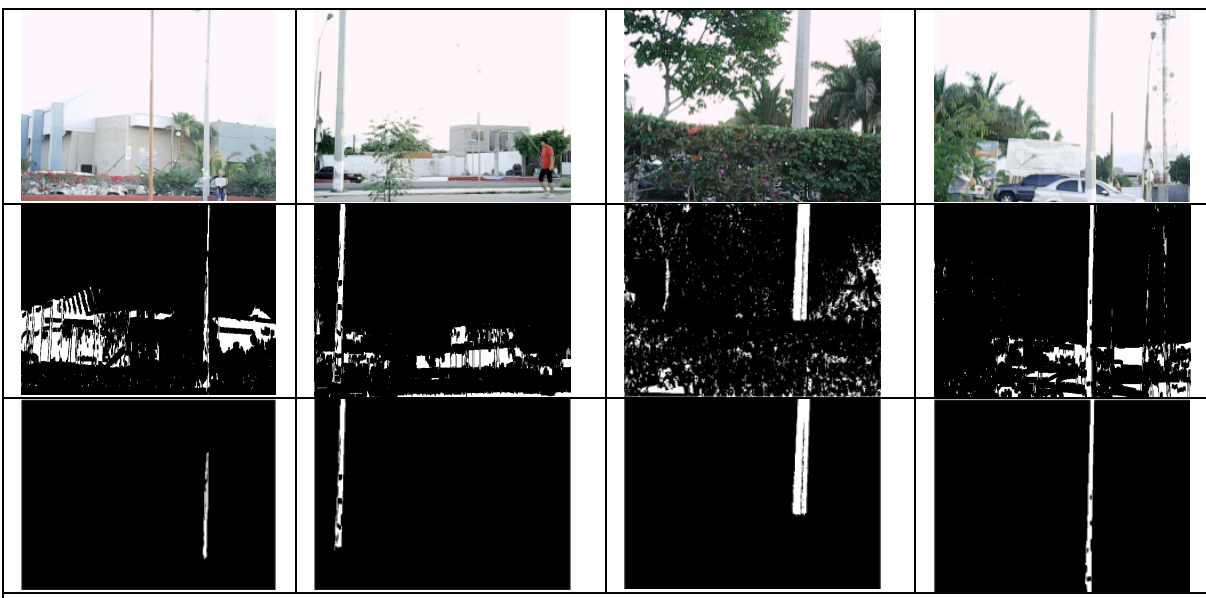

Fig. 8. The first row has the original photos, the next row has the color segmented images and the last has the segments identified by the Hu moments and Neural Network 
60 photos of different UP were used to test the proposed procedure with a $100 \%$ effectiveness with different illuminations, distance between camera an UP, inclinations and some natural occlusions by posters on the pole, trees, cars, signals and small graffiti's.

\section{Conclusions}

In this paper a methodology for electric pole recognition based on color, shape and photometric stereo vision was proposed. The system uses conventional and low cost cameras. Results were totally satisfactory with $100 \%$ effectiveness in range $5 \%$ to 95\% respect to $\mathrm{H}$ component of HSV scheme. The proposed method recognizes and locates utility poles. By comparing this method with the one proposed by [8] we can see the potential of this work. The proposed method was tested for recognition of utility poles in real conditions such as occlusions (from posters, graffiti's, trees and photos of partial poles), solar illumination, and geometrical distortions of the target. The effectiveness obtained from experiments, which indicates that the method is suitable for field applications.

\section{References}

[1] He, Y., Tatsuno, K.: An Example of Open Robot Controller Architecture - For Power Distribution Line Maintenance Robot System. World Academy of Science, Engineering and Technology 29, 266-271 (2008)

[2] Nakajima, C.: Automatic recognition of facility drawings and street maps utilizing the facility management database. In: Proceedings of the Third International Conference on Document Analysis and Recognition, vol. 1, pp. 516-519 (1995)

[3] Cetin, B.: Automated electric utility pole detection from aerial images. In: SOUTHEASTCON 2009, pp. 44-49. IEEE (2009)

[4] Igor, K., Amit, A., Ehud, R.: Color Invariants for Person Reidenti- fication. IEEE Transactions on Pattern Analysis and Machine Intelligence 35(7), 1622-1634 (2013)

[5] Berwick, D., Lee, S.: A Chromaticity Space for Specularity, Illumination Color and Illumination Pose-Invariant 3-D Object Recognition. In: Proc. IEEE Intl Conf. Computer Vision, pp. 165-170 (1998)

[6] Halawani, S.M., Sunar, M.S.: Interaction between Sunlight and the Sky Colour with 3D Objects in the Outdoor Virtual Environment. In: Proceedings of the 2010 Fourth Asia International Conference on Mathematical/Analytical Modelling and Computer Simulation, AMS 2010, pp. 470-475 (2010)

[7] Batllea, J., Casalsb, A., Freixeneta, J., Mart1, J.: A review on strategies for recognizing natural objects in colour images of outdoor scenes. Image and Vision Computing 18, 515-530 (2000)

[8] Yokoyama, H., Date, H., Kanai, S., Takeda, H.: Pole-like objects recognition from mobile laser scanning data using smoothing and principal component analysis. In: ISPRS Workshop, Laser scanning 2011, ISPRS, vol. XXXVIII, pp. 115-121 (2011)

[9] Peyton, Z., Peebles Jr.: Probability, Random Variables, and Random Signal Principles, pp.77-84. McGraw Hill (2000) 
[10] Prewitt, J.M.S.: Object Enhancement and Extraction in Picture processing and Psychopictorics. Academic Press (1970)

[11] Hu, M.K.: Visual Pattern Recognition by Moment Invariants. IRE Trans. Info. Theory IT8, 179-187 (1962)

[12] Lowe, D.G.: Three-dimensional object recognition from single two-dimensional images. Artificial Intelligence 31(3), 355-395 (1987)

[13] Barranco, A.I., Medel, J.: Automatic object recognition based on dimensional relation. Computación y Sistemas Journal 15(2), 267-272 (2011)

[14] Barranco, A.I., Medel, J.: Artificial vision and identification for intelligent orientation using a compass. Revista Facultad de Ingeniería Universidad de Antioquia, Rev. Fac. Ing. Univ. Antioquia N 58, 191-198 (2011)

[15] Zhang, Z.: A flexible new technique for camera calibration. IEEE Transactions on Pattern Analysis and Machine Intelligence 22(11), 1330-1334 (2000)

[16] Rodríguez, G.J., Gómez, J.L., Barranco, A.I., Martínez, S., Sandoval, J.: Visual 3D object recognition and location for manipulator robot. In: Proceedings of CIRC 2013, pp. 217-222 (2013)

[17] Burger, W., Burge, M.J.: Digital Image Processing: An Algorithmic Introduction Using Java, pp. 260-265. Springer (2010) 